\title{
A Novel Adaptive Frequency Estimation Algorithm Based on Interpolation FFT and Improved Adaptive Notch Filter
}

\author{
Shen Ting-ao ${ }^{1,2}$, Li Hua-nan ${ }^{1}$, Zhang Qi-xin ${ }^{1}$, Li Ming ${ }^{1}$ \\ ${ }^{1}$ Department of Petroleum Supply Engineering, Logistical Engineering University, Chongqing 401311, China \\ ${ }^{2}$ Corresponding Email: 279522560@qq.com
}

\begin{abstract}
The convergence rate and the continuous tracking precision are two main problems of the existing adaptive notch filter (ANF) for frequency tracking. To solve the problems, the frequency is detected by interpolation FFT at first, which aims to overcome the convergence rate of the ANF. Then, referring to the idea of negative feedback, an evaluation factor is designed to monitor the ANF parameters and realize continuously high frequency tracking accuracy. According to the principle, a novel adaptive frequency estimation algorithm based on interpolation FFT and improved ANF is put forward. Its basic idea, specific measures and implementation steps are described in detail. The proposed algorithm obtains a fast estimation of the signal frequency, higher accuracy and better universality qualities. Simulation results verified the superiority and validity of the proposed algorithm when compared with original algorithms.
\end{abstract}

Keywords: Adaptive notch filter, frequency estimation, Fast Fourier Transform, negative feedback.

\section{INTRODUCTION}

Frequency is a basic parameter to describe the characteristic of the signal, which has been widely used in many fields such as power systems, flow measurement, and fault diagnosis.

In the past three decades, lots of frequency estimation algorithms have been proposed to provide good performance, such as FFT [1], wavelet transform [2], correlation [3], ANF [4]-[5], and so on. Compared with other frequency estimation algorithms, ANF can automatically adjust the parameters according to the measured signal characteristics, and realize the estimation and tracking of frequency. It is a research hotspot of the current frequency estimation algorithm [6]-[7]. But the algorithm is more sensitive to the initial parameter value, and it is difficult to balance the convergence rate and the long tracking precision.

To resolve the above problems, a novel adaptive frequency estimation algorithm based on interpolation FFT and improved ANF is proposed. In Section 2, the ANF is introduced and analyzed. In Section 3, the proposed algorithm is elaborated, including the interpolation FFT algorithm which is introduced to improve the short time signal frequency estimation, and the improved ANF which is used to monitor and track signal frequency for long time, by designing an evaluation factor to adjust the ANF parameters using the feedback control principle. In Section 4, the proposed algorithm is validated by simulations. Finally, Section 5 concludes.

\section{ANALYSIS OF THE ADAPTIVE NOTCH FILTER}

\section{A. Principle of the adaptive notch filter}

Adaptive notch filter (ANF) is used to obtain the filter parameters, which could automatically adjust the filter parameters of the current time, and adapt to the unknown changes of the signal and noise statistical characteristics. The frequency characteristic of the ideal notch filter equals to 1 , and the other equals 0 . ANF can make the notch frequency automatically follow the changing of the input signal frequency. It can be used to eliminate the interference and to detect the sinusoidal signal in noise frequency [8]. The realization of the ANF is shown in Fig.1.

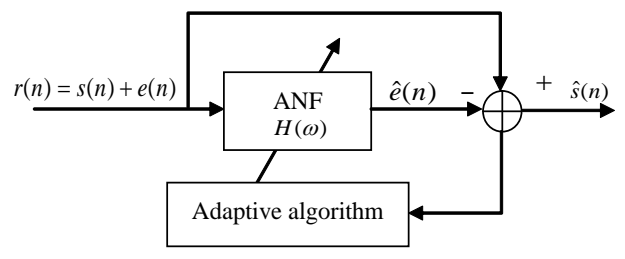

Fig.1. Structure of adaptive notch filter.

As shown in Fig.1., when an observation signal $r(n)$ gets through the ANF, sinusoidal signal $s(n)$ can be filtered, and the optimal estimation noise signal $\hat{e}(n)$ can be obtained, and then, by subtracting $\hat{e}(n)$ from the observed signal $r(n)$, the optimal estimation value $\hat{s}(n)$ can be obtained. 


\section{B. Problem analysis}

\section{1) Convergence rate}

According to the principle of ANF, if the input signal's a priori is unknown, the initial notch bandwidth tends to be larger, so that the signal frequency can be captured as soon as possible. But if the initial notch bandwidth is too wide, it will cause less filtered noise and lower accuracy of frequency estimation. With the adaptive adjustment of ANF parameters, the ANF will be gradually locked on the correct frequency, the notch bandwidth will be gradually reduced, the filtered noise will be higher, and the precision of frequency tracking will be much higher. The process of ANF from the beginning to locking on the correct frequency can be regarded as a convergence process, and the frequency estimation accuracy in the process is low.

In order to improve the precision of the frequency estimation in the convergence process of ANF, some frequency estimation methods can be used to improve the accuracy in the convergence process.

\section{2) Continuous tracking problem}

When the ANF is used to track the unknown signal frequency, to capture the signal frequency as quickly as possible, the initial notch bandwidth often sets to be larger. With the adaptive adjustment of ANF parameters, the convergence factor approaches to 1 , the notch bandwidth approaches to 0 , and the accuracy of the frequency estimation will be higher and higher. When the notch bandwidth approaches 0 , if the signal frequency changes and jumps out of the notch bandwidth, it will cause the notch in false frequency, and then cause larger frequency estimation error.

In the process of frequency tracking, along with the continuous adjustment of ANF parameters, the notch bandwidth will be gradually reduced. If the notch bandwidth approaches 0 , it will easily lead to the correct frequency out of the notch bandwidth, so that the ANF cannot perceive the change of the signal frequency, and then a larger frequency estimation error will be caused, which can be regarded as the tracking problem for long time.

To improve the frequency tracking estimation accuracy for long time, when the notch bandwidth approaches 0 , an evaluation factor can be set up to monitor and adjust ANF parameters in real-time, which aims to ensure the frequency value in notch bandwidth range and prevent the ANF loss of adaptive ability. All the aforementioned problems can be improved by adjusting the notch bandwidth.

\section{PROPOSED ALGORITHMS}

\section{A. Basic ideas}

From the above analysis, it is difficult to balance the convergence rate and the continuous tracking precision. Therefore, a novel frequency tracking algorithm based on interpolation FFT and improved ANF is put forward in this paper. Firstly, interpolation FFT algorithm is used to estimate the initial signal frequency, and the estimated value is set as the initial frequency before the ANF is stable (assuming a stable point of the ANF at M), and then, the improved ANF is used to estimate the signal frequency after M. M is an empirical value which could be obtained by lots of simulations, and it often sets among 500 1000.

\section{B. Concrete measure}

1) Convergence problem

In order to overcome the problem of the convergence rate of ANF, the interpolation FFT algorithm is used to estimate the short time frequency of the signal as follows.

Set the single frequency sine signal as follows

$$
s(t)=A \cos \left(2 \pi f_{0} t+\theta_{0}\right)
$$

where $\boldsymbol{A}, f_{0}$ and $\theta_{0}$ denote the amplitude, frequency, and initial phase of the signal, respectively. Generally, $f_{0}$ is expressed as:

$$
f_{0}=\left(k_{0}+\delta\right) \cdot f_{d}
$$

where $k_{0}$ denotes positive integer, $\delta$ denotes leakage errors and $|\delta| \leq 0.5, f_{d}$ denotes frequency resolution and $f_{d}=f_{s} / N, f_{s}$ denotes sampling frequency, $N$ denotes sampling number. The sampled signal can be illustrated as:

$$
s(n)=A \cos \left[2 \pi\left(k_{0}+\delta\right) \cdot n / N+\theta_{0}\right]
$$

The frequency spectrum $S(k)$ can be obtained by DFT

$$
S(k)=\frac{A \cdot \sin \left[\pi\left(k-f_{0} T\right)\right]}{2 \sin \left[\pi\left(k-f_{0} T\right) / N\right]} \cdot e^{j\left[\theta_{0}-\frac{N-1}{N}\left(k-f_{0} T\right) \pi\right]}
$$

where $T$ is the sampling time, and $f_{d}=f_{s} / N=1 / T=\Delta f$. Assuming $k_{1}=\operatorname{int}\left[f_{0} T\right]$ is the maximum amplitude point at $S(k)$. The maximum amplitude value $A_{1}$ can be expressed as:

$$
A_{1}=\left|S\left(k_{1}\right)\right|=\frac{N A \sin (\pi \delta)}{2 \pi \delta}
$$

The line of the relatively large amplitude next to $k_{1}$ is called second-rate amplitude value [9]. Assume the discrete frequency index value is $k_{2}$, the second-rate amplitude value $A_{2}$ can be approximated as:

$$
\begin{gathered}
A_{2}=\left|S\left(k_{2}\right)\right|=\frac{N A \sin (\pi \delta)}{2 \pi(1-|\delta|)} \\
\alpha=\frac{A_{2}}{A_{1}}=\frac{\delta}{1-|\delta|}
\end{gathered}
$$




$$
|\delta|=\frac{\alpha}{1+\alpha}=\frac{A_{2}}{A_{1}+A_{2}}
$$

According to the value of $\delta$, the estimated higher accuracy frequency value of $\hat{f}_{\mathrm{o}}$ can be obtained.

$$
\hat{f}_{0}=\left(k_{1} \pm|\delta|\right) \Delta f
$$

In equation (9), the symbol is dependent on the position of $k_{2}$. If $k_{2}=k_{1}+1$, it gets plus sign. If $k_{2}=k_{1}-1$, it gets minus sign.

\section{2) Long time tracking problem}

By using the negative feedback control principle, the parameters of ANF can be monitored and adjusted in realtime by setting an evaluation factor and the algorithm can be illustrated as follows.

As shown in Fig.1., the enhanced signal $\hat{s}(n)$ is independent of the noise $z(n)$. If the ANF works normally, the enhanced signal $\hat{s}(n)$ is significantly correlated with the initial input signal $r(n)$. According to the correlation, an evaluation factor $h(n)$ can be designed to detect whether the ANF is an effective tracking signal frequency or not. The structures of the improved ANF are shown in Fig.2.

The value of $h(n)$ can be obtained from the extra LMS block algorithm by the following:

$$
\left\{\begin{array}{l}
\varepsilon(n)=\hat{s}(n)-h(n) r(n) \\
h(n)=h(n-1)+\mu_{h} \varepsilon(n) r(n)
\end{array}\right.
$$

where $\mu_{h}$ is the step size, $\boldsymbol{h}$ is deduced from the WienerHopf equation in [10].

$$
\begin{aligned}
& h \cdot E\left\{r^{2}(n)\right\}=E\{r(n) \hat{s}(n)\} \\
\Leftrightarrow & h \cdot E\left\{[s(n)+e(n)]^{2}\right\}=E\{[s(n)+e(n)] \hat{s}(n)\} \\
\Leftrightarrow & \left.h \cdot\left(E\left\{s^{2}(n)\right\}+E\left\{e^{2}(n)\right]\right\}\right)=E\{s(n) \hat{s}(n)\}+E\{e(n)\} E\{\hat{s}(n)\} \\
\Leftrightarrow & h=\frac{E\{s(n) \hat{s}(n)\}}{A^{2} / 2+\sigma_{e}^{2}}
\end{aligned}
$$

where $E\{s(n) e(n)\}=0, E\{e(n)\}=0, E\left\{e^{2}(n)\right\}=\sigma_{e}^{2}, E\left\{s^{2}(n)\right\}=A^{2} / 2$.

If the ANF locks on the right frequency, then $\hat{s}(n) \approx s(n)$, and $h$ converges to

$$
h=\frac{A^{2} / 2}{A^{2} / 2+\sigma_{e}^{2}}=\frac{A^{2}}{A^{2}+2 \sigma_{e}^{2}}
$$

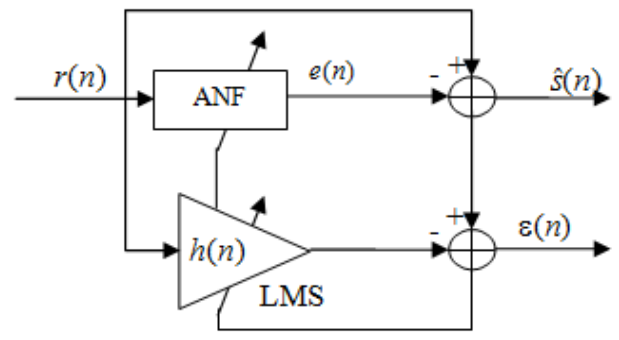

Fig.2. Structures of the improved adaptive notch filter.

If the ANF locks on the wrong frequency, then $\hat{s}(n) \approx e(n)$, substitute it into equation (11), and $h$ converges to 0 since $E\{s(n) \hat{s}(n)\}=0$. Therefore, the evaluation factor $h(n)$ can be used to judge whether the ANF effectively tracks the signal frequency or not. If the $h(n)$ falls below the set value $T_{h}$, the ANF can resume the parameters to assure the notch bandwidth contains the signal frequency again.

According to the above basic ideas, the improved ANF algorithm is illustrated in Fig.3.

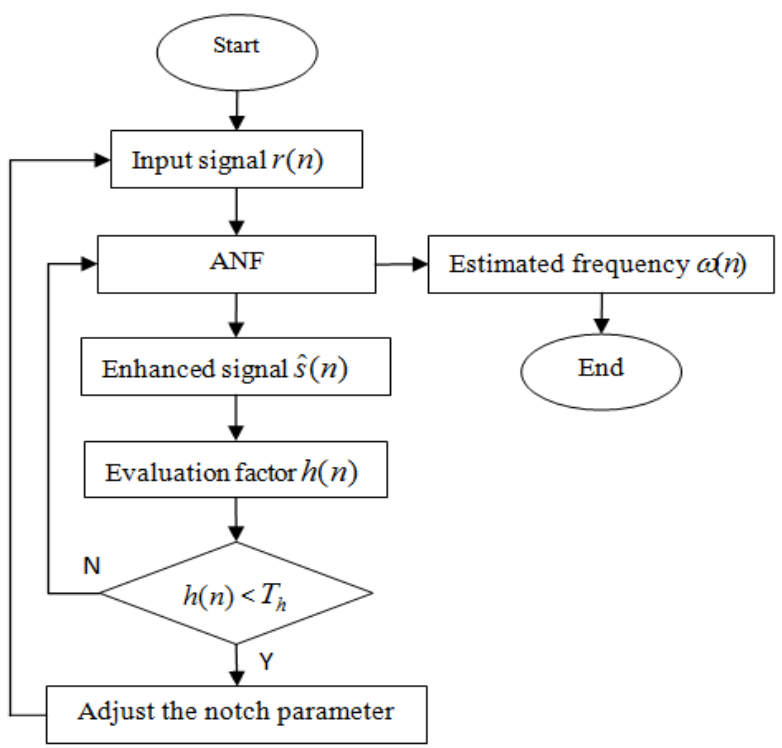

Fig.3. Flow chart of the improve ANF.

The overall block diagram of the proposed technique is summarized in Fig.4.

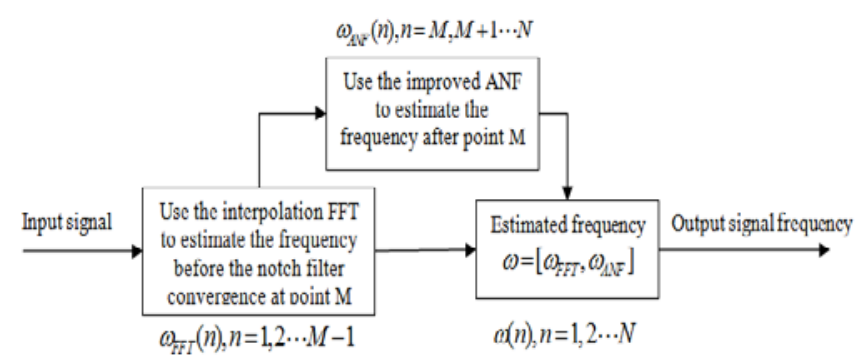

Fig.4. Flow chart of the proposed frequency estimation algorithm. 
As shown in Fig.3. and Fig.4., the proposed algorithm obtains fast frequency tracking, long time frequency tracking and high accuracy estimation characteristics even for the unknown signal.

\section{SimUlation ANALYSES}

In order to verify the validity and the universality of the proposed algorithm, the algorithm is applied in lattice adaptive notch filter (L-ANF) [11], simplified lattice adaptive notch filter (SL-ANF) [12], and SMM adaptive notch filter (SMM-ANF) [13], respectively. In simulations, the input signal model and ANF parameters refer to references [11]-[13], and all the ANF parameters remain unchanged. The proposed algorithm just introduces the FFT algorithm at the beginning of the frequency estimation, and adds an evaluation factor in ANF for long-time frequency estimation, the parameters $T_{h}=0.05$ and $\mu_{h}=0.008$.

Fig.5. shows the estimated frequencies by original L-ANF and the improved algorithm in this paper based on L-ANF. Fig.6. shows the estimated frequencies by original SL-ANF and the improved algorithm in this paper based on SL-ANF. Fig.7. shows the estimated frequencies by original SMMANF and the improved algorithm in this paper based on SMM-ANF. In order to validate the universality of the proposed method in this paper, the curves of Fig.5. to Fig.7. are randomly generated.

As shown in Fig.5. to Fig.7., which include L-ANF, SLANF and SMM-ANF, there are convergence process and long precision problem when tracking signal frequencies. Compared with the original algorithm, the improved algorithm of frequency tracking curve is better and closer to the true frequency. The proposed algorithm maintains higher estimation accuracy in the whole process of frequency tracking, which overcomes the influence of the convergence process, and solves the problem of long time tracking. In addition, we can see from Fig.5. to Fig.7., that the proposed algorithm can be applied to many kinds of ANF, and all the randomly generated curves maintain a good tracking effect, which verifies the universality of the proposed algorithm.

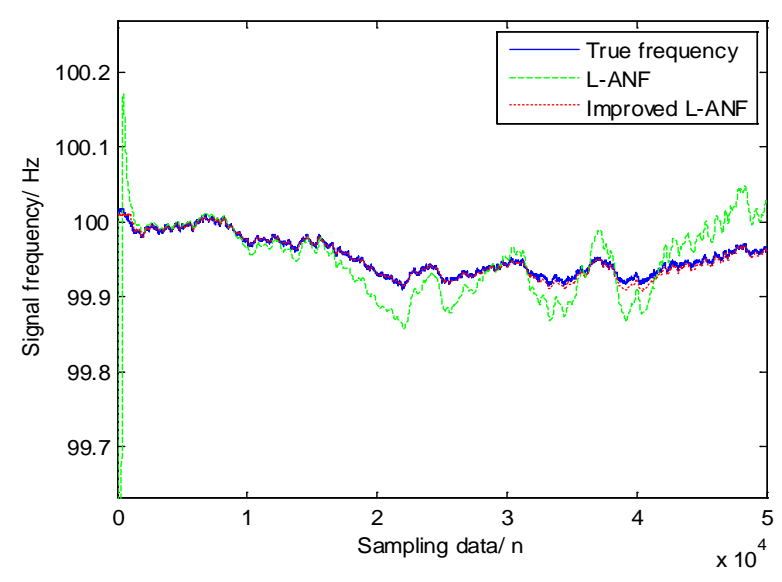

Fig.5. Comparisons by L-ANF and proposed method.

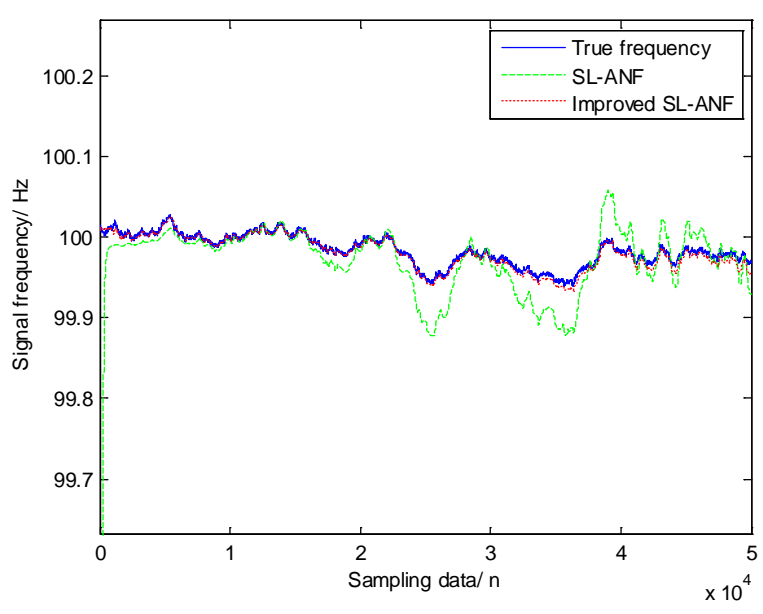

Fig.6. Comparisons by SL-ANF and proposed method.

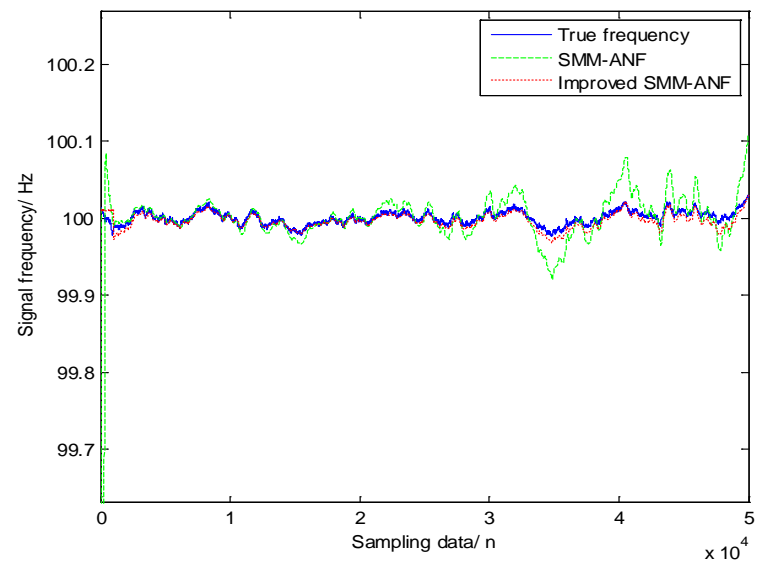

Fig.7. Comparisons by SMM-ANF and proposed method.

Testing 100 independent and random experiments, the mean of square errors (MSE) of the three types of ANF is compared and calculated according to the equation (13), which is shown in Table 1 . To avoid the influence of convergence of the ANF algorithms, the signals after 1000th point are intercepted to calculate the frequency.

$$
M S E=\frac{1}{49000} \sum_{i=1001}^{50000}[\hat{\omega}(i)-\omega(i)]^{2}
$$

As shown in Table 1., the MSE of the proposed algorithm is smaller than the original algorithm, which also demonstrates the superior performance of the proposed algorithm.

Table 1. Comparisons of MSE acquired by three types of ANF.

\begin{tabular}{|l|c|c|}
\hline \multirow{2}{*}{ ANF } & \multicolumn{2}{|c|}{ MSE } \\
\cline { 2 - 3 } & Original algorithm & Proposed algorithm \\
\hline L-ANF & $3.72 \times 10^{-7}$ & $1.66 \times 10^{-8}$ \\
\hline SL-ANF & $3.57 \times 10^{-7}$ & $1.65 \times 10^{-8}$ \\
\hline SMM-ANF & $3.19 \times 10^{-7}$ & $1.64 \times 10^{-8}$ \\
\hline
\end{tabular}




\section{CONCLUSIONS}

In order to solve the problem of convergence rate and long time frequency tracking, a new frequency estimation algorithm based on interpolation FFT and improved ANF is proposed. Simulations show that the proposed algorithm obtains the following characteristics:

1) Using interpolation FFT algorithm in the initial stage of signal frequency estimation, which can improve the convergence process of frequency estimation accuracy, at the same time, the estimate value by interpolation FFT algorithm can be used as the initial frequency, which could speed up the convergence rate of the ANF.

2) Using the negative feedback control theory to design the evaluation factor, it can effectively solve the problem of long time frequency tracking of the ANF.

3) The proposed algorithm is more universal, which can apply to other types of ANF.

For future research, we will focus on extending and generalizing this type of algorithms to a more general system identification scheme, and further research is under discussion.

\section{ACKNOWLEDGMENT}

This work was supported by National Natural Science Foundation of China (Grant Nos. 61601493) and Science Foundation of Logistical Engineering University (Grant Nos. YQ16-420804). The authors would like to thank the Associate Editor and the reviewers for their helpful comments, which improved the quality of this paper.

\section{REFERENCES}

[1] Chen, K., Wang, J., Zhang, S. (2008). Spectrum correction based on the complex ratio of discrete spectrum around the main-lobe. Journal of Vibration Engineering, 21 (3), 314-318.

[2] Wang, X., Xu, K. (2005). Fundamental wave extraction and frequency measurement base on wavelet transform. Chinese Journal of Scientific Instrument, 26 (2), 146-151.

[3] Ksibi, R.E., Besbes, H., Valcarce, R.L., Cherif, S. (2010). Frequency estimation of real-valued singletone in colored noise using multiple autocorrelation lags. Signal Processing, 90 (7), 2303-2307.

[4] Loetwassana, W., Punchalard, R., Koseeyaporn, J., Wardkein, P. (2012). Unbiased plain gradient algorithm for a second-order adaptive IIR notch filter with constrained poles and zeros. Signal Processing, 90 (8), 2513-2520.

[5] Punchalard, R. (2012). Mean square error analysis of unbiased modified plain gradient algorithm for secondorder adaptive IIR notch filter. Signal Processing, 92 (11), 2815-2820.

[6] George, N., Anthony, T. (2010). Application of adaptive lattice filters for modal parameter tacking of a single flexible link carrying a shifting payload. Mechanical Systems and Signal Processing, 24 (5), 1338-1348.

[7] Regalia, P.A. (2010). A complex adaptive notch filter. IEEE Signal Processing Letters, 17 (11), 937-940.

[8] Cho, N.I., Choi, C.H., Lee, S.U. (1989). Adaptive line enhancement by using an IIR lattice notch filter. IEEE Transactions on Acoustics, Speech and Signal Processing, 37 (4), 585-589.

[9] Shen, T., Tu, Y., Li, M., Zhang, H. (2015). A new phase difference measurement algorithm for extreme frequency signals based on discrete time Fourier transform with negative frequency contribution. Review of Scientific Instruments, 86 (1), 015104.

[10] Ta, M., Thai, H., DeBrunner, V. (2009). Stochastic search methods to improve the convergence of adaptive notch filter. In IEEE 13th Digital Signal Processing Workshop, January 4-7, 2009. IEEE, 78-83.

[11] Xu, K., Ni, W., Chen, Z. (2006). A signal processing method for Coriolis mass flowmeter based on timevarying signal model and lattice notch filter. Chinese Journal of Scientific Instrument, 27 (2), 596-601.

[12] Ni, W., Xu, K. (2007). A signal processing method for Coriolis flowmeter based on time-varying signal model and normalized lattice notch filter. Acta Metrologica Sinica, 28 (3), 243-247.

[13] Tu, Y., Su, F., Shen, T., Zhang, H. (2011). Frequency tracking method and simulation for Coriolis Mass Flowmeter based on new adaptive notch filter. Journal of Chongqing University, 34 (10), 147-152.

Received October 26, 2016. Accepted February 2, 2017. 